PROCEEDINGS OF THE

AMERICAN MATHEMATICAL SOCIETY

Volume 140, Number 5, May 2012, Pages 1777-1783

S 0002-9939(2011)11084-0

Article electronically published on September 7, 2011

\title{
A NOTE ON THE HITCHIN-THORPE INEQUALITY AND RICCI FLOW ON 4-MANIFOLDS
}

\author{
YUGUANG ZHANG AND ZHENLEI ZHANG \\ (Communicated by Michael Wolf)
}

\begin{abstract}
In this short paper, we prove a Hitchin-Thorpe type inequality for closed 4-manifolds with non-positive Yamabe invariant and admitting long time solutions of the normalized Ricci flow equation with bounded scalar curvature.
\end{abstract}

\section{INTRODUCTION}

A Riemannian metric $g$ on a smooth manifold $M$ is called an Einstein metric if

$$
\operatorname{Ric}_{g}=c g
$$

where $R i c_{g}$ is the Ricci tensor and $c$ is a constant. If a closed oriented 4-manifold $M$ admits an Einstein metric $g$, there is an inequality, the Hitchin-Thorpe inequality, for the Euler number $\chi(M)$ and the signature $\tau(M)$ of $M$ :

$$
2 \chi(M)-3|\tau(M)| \geq 0
$$

(cf. [10] or Theorem 6.35 in [3]). This inequality serves as a topological obstruction for the existence of Einstein metrics on 4-manifolds; i.e. if (1.1) is not satisfied, then $M$ would not admit any Einstein metric.

The Ricci flow was introduced by Hamilton in 8 to find Einstein metrics on a given manifold, which is the following evolution equation for a smooth family of metrics $g(t), t \in[0, T)$ :

$$
\frac{\partial}{\partial t} g(t)=-2 R i c_{t}
$$

The normalized Ricci flow is

$$
\frac{\partial}{\partial t} g(t)=-2 R i c_{t}+\frac{2 r(t)}{n} g(t)
$$

where $r(t)=\frac{\int_{M} R_{t} d v_{g(t)}}{\operatorname{Vol}_{g(t)}(M)}$ denotes the average scalar curvature and $R_{t}$ denotes the scalar curvature of $g(t)$. The normalized Ricci flow is just a transformation of (1.2) by rescaling the space and time such that the volume preserves to be a constant along the flow. If (1.3) admits a long time solution $g(t), t \in[0, \infty)$, and $g(t)$ converges to a Riemannian metric $g_{\infty}$ on $M$ in some suitable sense, when $t \rightarrow \infty$,

Received by the editors May 26, 2010 and, in revised form, January 23, 2011.

2010 Mathematics Subject Classification. Primary 53C44.

The first author was supported by NSFC-10901111 and KM-210100028003

The second author was supported by NSFC-09221010056.

(C)2011 American Mathematical Society Reverts to public domain 28 years from publication 
then $g_{\infty}$ is an Einstein metric or a Ricci-soliton. However, (1.3) may not admit any long time solution, and even if there is such a solution, $g(t)$ may not converge to a Riemannian metric on $M$. It is expected that the inequality (1.1) is also a topological obstruction for the existence of long time solutions of (1.3) on 4manifolds, at least for 4-manifolds with a non-positive Yamabe invariant. In [6], an analog inequality of (1.1) was obtained for long time solutions of (1.3) under some hypothesis. In this paper, we will continue to study the Hitchin-Thorpe type inequality for 4-manifolds admitting long time solutions of (1.3).

For a closed Riemannian $n$-manifold $(M, g)$ and a function $f \in C^{\infty}(M)$, set

$$
\mathcal{F}(g, f)=\int_{M}\left(R_{g}+|\nabla f|^{2}\right) e^{-f} d v_{g} .
$$

The Perelman $\lambda$-functional is defined by

$$
\lambda_{M}(g)=\inf _{f}\left\{\mathcal{F}(g, f) \mid \int_{M} e^{-f} d v_{g}=1\right\},
$$

which is the lowest eigenvalue of the operator $-4 \triangle+R_{g}$. Perelman [15] has established the monotonicity property of $\bar{\lambda}_{M}(g(t))=\lambda_{M}(g(t)) \mathrm{Vol}_{g(t)}(M)^{\frac{2}{n}}$ along the Ricci flow. A diffeomorphism invariant $\bar{\lambda}_{M}$ of $M$ is defined (cf. [16], [12]) by

$$
\bar{\lambda}_{M}=\sup _{g \in \mathcal{M}} \bar{\lambda}_{M}(g)
$$

where $\mathcal{M}$ is the set of Riemannian metrics on $M$. By [1 and 2], $\bar{\lambda}_{M}$ is equal to the classical Yamabe invariant whenever $\bar{\lambda}_{M} \leq 0$ or the Yamabe invariant is non-positive.

In 6] it was proved that if $g(t), t \in[0, \infty)$, is a solution to (1.3) with $|R(g(t))|<C$ for a constant $C$ independent of $t$ on a closed oriented 4-manifold $M$ with $\bar{\lambda}_{M}<0$, then

$$
2 \chi(M)-3|\tau(M)| \geq \frac{1}{96 \pi^{2}} \bar{\lambda}_{M}^{2}>0 .
$$

Based on this inequality and the Seiberg-Witten theory, Ishida [11] recently showed that the existence of a long time non-singular solution really depends on the smooth structure of the underlying manifold. The goal of the present paper is to relax the assumption $\bar{\lambda}_{M}<0$ to $\bar{\lambda}_{M} \leq 0$.

Theorem 1.1. Let $M$ be a closed oriented 4-manifold with $\bar{\lambda}_{M} \leq 0$. If $M$ admits a long time solution $g(t), t \in[0, \infty)$, of (1.3) with scalar curvature $\left|R_{t}\right|<C$ for a constant $C$ independent of $t$, then

$$
2 \chi(M)-3|\tau(M)| \geq \frac{1}{96 \pi^{2}} \bar{\lambda}_{M}^{2}
$$

The hypothesis of $\bar{\lambda}_{M} \leq 0$ holds for many cases, for example, complex projective surfaces with non-negative Kodaira dimension by [14], which include K3-surfaces, projective surfaces of general type and some surfaces of elliptic type, etc. Furthermore, Theorem 2 in [13] shows that if $M$ is a closed oriented 4-manifold with a monopole class $c_{1}(\mathfrak{c})$ that is not a torsion class and satisfies $c_{1}^{2}(\mathfrak{c}) \geq 0$, then

$$
\bar{\lambda}_{M} \leq-\sqrt{32 \pi^{2} c_{1}^{2}(\mathfrak{c})} \leq 0
$$


(see also [5] for the case of $c_{1}^{2}(\mathfrak{c})>0$ ). In [6], a Miyaoka-Yau type inequality was proved for 4-manifolds with a monopole class $c_{1}(\mathfrak{c})$ such that $c_{1}^{2}(\mathfrak{c})>0$ and admitting long time solutions of (1.3) with bounded scalar curvature.

The assumption of bounded scalar curvature is a technique assumption, and we hope that it can be removed in future study. However, it can be verified for some cases. In 4, it was shown that a K3-surface $M$ admits a long time solution $g(t)$, $t \in[0, \infty)$, of (1.3) and that $g(t)$ converges to a Ricci-flat Kähler-Einstein metric on $M$. Thus the scalar curvature of $g(t)$ is uniformly bounded. Since $\bar{\lambda}_{M}=0$ (cf. [14]), Theorem 1.1 can apply to this case. If $M$ is a complex minimal projective surface of elliptic type with Kodaira dimension 1 and no singular fibers, then $\bar{\lambda}_{M}=0$ by 14 and $M$ admits long time solutions $\tilde{g}(\tilde{t}), \tilde{t} \in[0, \infty)$, of the following Kähler-Ricci flow equation:

$$
\frac{\partial}{\partial \tilde{t}} \tilde{g}(\tilde{t})=-\operatorname{Ric}_{\tilde{t}}-\tilde{g}(\tilde{t})
$$

by [19] and 20]. By Corollary 1.1 in [18, the scalar curvature of $\tilde{g}(\tilde{t})$ is uniformly bounded along (1.4). A straightforward calculation would show that a transformation of $\tilde{g}(\tilde{t}), \tilde{t} \in[0, \infty)$, by rescaling the metric and time, gives a long time solution of (1.3) with bounded scalar curvature. Thus the assumption of Theorem 1.1 is satisfied. If $M$ is a complex minimal projective surface of general type, which satisfies $\bar{\lambda}_{M}<0$ by [14, then $M$ admits long time solutions of (1.3) with bounded scalar curvature by [4, 19, 20, 23] and the same arguments as above. In 21, an alternative proof of the Miyaoka-Yau inequality for minimal projective manifolds of general type was obtained by using the result in [23] and a similar Ricci flow argument.

An analog Hitchin-Thorpe type inequality for non-compact 4-manifolds admitting non-singular solutions of (1.3) is obtained in [7. We assume $\operatorname{Vol}_{g(t)}(M) \equiv 1$ in this paper for convenience. We shall prove Theorem 1.1 in the next section.

\section{Proof of Theorem 1.1}

The goal of this section is to prove Theorem 1.1 which essentially depends on the following estimate for the volume along Ricci flow in [22].

Lemma 2.1 (Lemma 3.1 in [22]). Let $\bar{g}(\bar{t}), \bar{t} \in[0, T)$, be a solution to the Ricci flow equation (1.2), i.e.

$$
\frac{\partial}{\partial \bar{t}} \bar{g}(\bar{t})=-2 R i c_{\bar{t}}
$$

on a closed manifold $M$. If $\lambda_{M}(\bar{g}(\bar{t})) \leq 0$, for all $\bar{t}$, then there exist constants $c_{1}, c_{2}>0$ depending only on $\bar{g}(0)$ such that, for all $\bar{t} \geq 0$,

$$
\operatorname{Vol}_{\bar{g}(\bar{t})}(M) \geq c_{1} e^{-c_{2} \bar{t}}
$$

Because of the importance of this lemma, we present the sketch of the proof here for the reader's convenience.

Sketch of the proof. First, we recall some basics about the $\mu$ functional introduced by Perelman [15. Given a closed Riemannian manifold $(M, g)$ and a function $f \in C^{\infty}(M)$ and a constant $\tau>0$, define

$$
\mathcal{W}(g, f, \tau)=\int_{M}\left[\tau\left(R_{g}+|\nabla f|^{2}\right)+f-n\right](4 \pi \tau)^{-n / 2} e^{-f} d v_{g}
$$


and then set

$$
\mu(g, \tau)=\inf \left\{\mathcal{W}(g, f, \tau) \mid \int_{M}(4 \pi \tau)^{-n / 2} e^{-f} d v_{g}=1\right\} .
$$

By a result of Rothaus [17, for each $\tau>0$, there is a smooth minimizer $f$ such that $\mu(g, \tau)=\mathcal{W}(g, f, \tau)$. In [22], some bounds of the $\mu$-functional were obtained. First, Lemma 2.1 in [22] shows that there is a lower bound for $\tau>\frac{n}{8}$,

$$
\mu(g, \tau) \geq \lambda_{M}(g) \tau-\frac{n}{2} \ln (4 \pi \tau)-n-\frac{n}{8}\left(\lambda_{M}(g)-\inf R_{g}\right)-n \ln C_{s},
$$

where $C_{s}$ denotes the Sobolev constant for $g$, i.e. $\|\phi\|_{L^{\frac{2 n}{n-2}(g)}} \leq C_{s}\|\phi\|_{H^{1,2}(g)}$ for all $\phi \in C^{\infty}(M)$. Second, Corollary 2.3 in 22] says that if $\lambda_{M}(g) \leq 0$, then

$$
\mu(g, \tau) \leq \ln \operatorname{Vol}_{g}(M)-\frac{n}{2} \ln (4 \pi \tau)-n+1 .
$$

In [15, Perelman proved the monotonicity of the $\mu$-functional along the Ricci flow:

Theorem 2.2 ([15]). Let $\bar{g}(\bar{t})$ be a solution to the Ricci flow equation (1.2) on a closed manifold $M$. Denote $\tau(\bar{t})=A-\bar{t}$ for some constant $A>0$. Then $\mu(\bar{g}(\bar{t}), \tau(\bar{t}))$ is non-decreasing whenever it makes sense.

Note that $\lambda_{M}(\bar{g}(\bar{t})) \leq 0$. Substituting $\tau=\frac{n}{8}$ into (2.3) and then using Theorem 2.2 and (2.2), we have

$$
\begin{aligned}
\operatorname{Vol}_{\bar{g}(\bar{t})}(M) & \geq \exp \left(\mu\left(\bar{g}(\bar{t}), \frac{n}{8}\right)+\frac{n}{2} \ln \left(\frac{n}{2} \pi\right)+n-1\right) \\
& \geq \exp \left(\mu\left(\bar{g}(0), \frac{n}{8}+\bar{t}\right)+\frac{n}{2} \ln \left(\frac{n}{2} \pi\right)+n-1\right) \\
& \geq \exp \left(\lambda_{M}(\bar{g}(0)) \bar{t}-\frac{n}{2} \ln \left(1+\frac{8}{n} \bar{t}\right)+\frac{n}{8} \inf R_{0}-n \ln C_{s}(\bar{g}(0))-1\right) \\
& \geq \exp \left(\left(\lambda_{M}(\bar{g}(0))-4\right) \bar{t}+\frac{n}{8} \inf R_{0}-n \ln C_{s}(\bar{g}(0))-1\right)
\end{aligned}
$$

We obtain the conclusion.

Now we can prove:

Lemma 2.3. Let $M$ be a closed n-manifold with $\bar{\lambda}_{M} \leq 0$ and let $g(t), t \in[0, \infty)$, be a long time solution of (1.3) with scalar curvature $\left|R_{t}\right|<C$ for a constant $C$ independent of $t$. We have

$$
\liminf _{t \rightarrow \infty} r(t)=\liminf _{t \rightarrow \infty} \int_{M} R_{t} d v_{g(t)} \leq 0 .
$$

Proof. If it is not true, there is a constant $\delta>0$ such that, for $t \gg 1, r(t)>\delta$. By a translation on $t$, we assume that $r(t)>\delta$ for all $t>0$.

Let $\bar{g}(\bar{t})=\sigma(t) g(t), \bar{t} \in[0, T)$, be the corresponding Ricci flow solution, i.e. $\frac{\partial}{\partial t} \bar{g}(\bar{t})=-2 \operatorname{Ric}_{\bar{t}}$ with $\bar{g}(0)=g(0)$, which implies $\sigma(t)=\exp \left(-\frac{2}{n} \int_{0}^{t} r(s) d s\right)$ and $\bar{t}=\int_{0}^{t} \sigma(s) d s$. Thus

$$
T=\int_{0}^{\infty} \sigma(s) d s=\int_{0}^{\infty} \exp \left(-\int_{0}^{s} \frac{2}{n} r(u) d u\right) d s<\frac{n}{2 \delta} .
$$


Now we can compute

$$
\begin{aligned}
(T-\bar{t}) \operatorname{Vol}_{\bar{g}(\bar{t})}(M)^{-\frac{2}{n}} & =\sigma(t)^{-1}(T-\bar{t}) \\
& =\exp \left(\int_{0}^{t} \frac{2}{n} r(u) d u\right) \cdot \int_{t}^{\infty} \exp \left(-\int_{0}^{s} \frac{2}{n} r(u) d u\right) d s \\
& \geq \int_{t}^{\infty} \exp \left(-\int_{t}^{s} \frac{2}{n} r(u) d u\right) d s \\
& \geq \int_{t}^{\infty} \exp \left(-\frac{2}{n} C(s-t)\right) d s \\
& \geq \frac{n}{2 C},
\end{aligned}
$$

by $r(t) \leq \sup \left|R_{t}\right| \leq C$ for a constant $C>0$ independent of $t$. Thus there exists $C_{1}<\infty$ such that

$$
\operatorname{Vol}_{\bar{g}(\bar{t})}(M) \leq C_{1}(T-\bar{t})^{n / 2}
$$

which implies that

$$
\lim _{\bar{t} \rightarrow T} \operatorname{Vol}_{\bar{g}(\bar{t})}(M)=0 .
$$

However, since $\bar{\lambda}_{M} \leq 0$, we have $\lambda_{M}(\bar{g}(\bar{t})) \leq 0$ and

$$
\operatorname{Vol}_{\bar{g}(\bar{t})}(M) \geq c_{1} e^{-c_{2} \bar{t}} \geq c_{1} e^{-c_{2} T}
$$

for two constants $c_{1}>0$ and $c_{2}>0$ by Lemma 2.1, which is a contradiction.

Before proving Theorem 1.1, we recall the evolution equations for volume forms and scalar curvatures along the normalized Ricci flow (1.3):

$$
\begin{gathered}
\frac{\partial}{\partial t} d v_{g(t)}=-\left(R_{t}-r(t)\right) d v_{g(t)} \quad \text { and } \\
\frac{\partial}{\partial t} R_{t}=\triangle_{t} R_{t}+2\left|R i c_{t}\right|^{2}+\frac{2}{n} R_{t}\left(R_{t}-r(t)\right),
\end{gathered}
$$

where $R i c_{t}=R i c_{t}-\frac{R_{t}}{n} g(t)$ denotes the Einstein tensor (cf. [9]).

Proof of Theorem 1.1. Note that

$$
\breve{R}_{t}=\inf _{M} R_{t} \leq \lambda_{M}(g(t)) \leq \bar{\lambda}_{M} \leq 0 .
$$

If $\breve{R}_{t} \leq-c<0$ for a constant $c>0$, we obtain the conclusion by Lemma 2.7 and Lemma 3.1 in $[$.

From the maximal principal,

$$
\frac{\partial}{\partial t} \breve{R}_{t} \geq \frac{1}{2} \breve{R}_{t}\left(\breve{R}_{t}-r(t)\right) \geq 0,
$$

and thus $\breve{R}_{t}$ is non-decreasing. Therefore, the only case left to prove is that of

$$
\lim _{t \longrightarrow \infty} \breve{R}_{t}=0 .
$$

By Lemma 2.3 and $\breve{R}_{t} \leq r(t)$,

$$
\liminf _{t \rightarrow \infty} r(t)=0,
$$

which implies that $\bar{\lambda}_{M}=0$. 
First, we assume that there is a sequence $t_{k}^{\prime} \longrightarrow \infty$ such that $r\left(t_{k}^{\prime}\right)>\epsilon$ for a constant $\epsilon>0$ independent of $k$. Since $\liminf _{t \rightarrow \infty} r(t)=0$, there are $t_{k} \in\left(t_{k}^{\prime}, t_{k+1}^{\prime}\right)$ such that, for $k \gg 1$,

$$
r\left(t_{k}\right)=\inf _{\left(t_{k}^{\prime}, t_{k+1}^{\prime}\right)} r(t) \longrightarrow 0, \quad \frac{d r}{d t}\left(t_{k}\right)=0 .
$$

Now, we assume $\lim _{t \rightarrow \infty} r(t)=0$. If $\left|\frac{d r}{d t}(t)\right|>\delta>0$ for a constant $\delta$ independent of $t$ when $t \gg 1,|r(t)-r(0)|>\delta t$, which is a contradiction. Thus there is a sequence $t_{k} \longrightarrow \infty$ such that

$$
\lim _{t_{k} \longrightarrow \infty} r\left(t_{k}\right)=0, \quad \lim _{t_{k} \longrightarrow \infty} \frac{d r}{d t}\left(t_{k}\right)=0 .
$$

In both cases, we have

$$
\begin{aligned}
0 & =\lim _{k \rightarrow \infty} \frac{d r}{d t}\left(t_{k}\right) \\
& =\lim _{k \rightarrow \infty} \int_{M}\left(2\left|R i c_{t_{k}}^{o}\right|^{2}-\frac{1}{2} R_{t_{k}}\left(R_{t_{k}}-r\left(t_{k}\right)\right)\right) d v_{g\left(t_{k}\right)} \\
& \geq \lim _{k \rightarrow \infty} \int_{M} 2\left|R i c_{t_{k}}^{o}\right|^{2} d v_{g\left(t_{k}\right)}-\lim _{k \rightarrow \infty} C \int_{M}\left|R_{t_{k}}-r\left(t_{k}\right)\right| d v_{g\left(t_{k}\right)} \\
& \geq \lim _{k \rightarrow \infty} \int_{M} 2\left|R i c_{t_{k}}^{o}\right|^{2} d v_{g\left(t_{k}\right)}-\lim _{k \rightarrow \infty} C \int_{M}\left(R_{t_{k}}+r\left(t_{k}\right)-2 \breve{R}_{t_{k}}\right) d v_{g\left(t_{k}\right)} \\
& =\lim _{k \rightarrow \infty} \int_{M} 2\left|R i c_{t_{k}}^{o}\right|^{2} d v_{g\left(t_{k}\right)}-\lim _{k \rightarrow \infty} 2 C\left(r\left(t_{k}\right)-\breve{R}_{t_{k}}\right) \\
& =\lim _{k \rightarrow \infty} \int_{M} 2\left|R i c_{t_{k}}^{o}\right|^{2} d v_{g\left(t_{k}\right)}
\end{aligned}
$$

by (2.7), (2.8), and the assumption $\left|R_{t}\right|<C$ for a constant $C$ independent of $t$.

The Chern-Gauss-Bonnet formula and the Hirzebruch signature theorem (cf. [3] show that, for any metric $g$ on $M$,

$$
\begin{gathered}
\chi(M)=\frac{1}{8 \pi^{2}} \int_{M}\left(\frac{R_{g}^{2}}{24}+\left|W_{g}^{+}\right|^{2}+\left|W_{g}^{-}\right|^{2}-\frac{1}{2}\left|R i c_{g}\right|^{2}\right) d v_{g} \quad \text { and } \\
\tau(M)=\frac{1}{12 \pi^{2}} \int_{M}\left(\left|W_{g}^{+}\right|^{2}-\left|W_{g}^{-}\right|^{2}\right) d v_{g}
\end{gathered}
$$

where $W_{g}^{+}$and $W_{g}^{-}$are the self-dual and anti-self-dual Weyl tensors respectively. Thus

$$
\begin{aligned}
2 \chi(M)-3|\tau(M)| & \geq \liminf _{k \rightarrow \infty} \frac{1}{4 \pi^{2}} \int_{M}\left(\frac{1}{24} R_{t_{k}}^{2}-\frac{1}{2}\left|R i c_{t_{k}}\right|^{2}\right) d v_{g\left(t_{k}\right)} \\
& =\liminf _{k \rightarrow \infty} \frac{1}{4 \pi^{2}} \int_{M} \frac{1}{24} R_{t_{k}}^{2} d v_{g\left(t_{k}\right)} \geq 0
\end{aligned}
$$

Since $\bar{\lambda}_{M}=0$ in this case, we obtain the conclusion.

\section{REFERENCES}

[1] K. Akutagawa, M. Ishida, and C. LeBrun, Perelman's invariant, Ricci flow, and the Yamabe invariants of smooth manifolds, Arch. Math. 88 (2007), 71-76. MR2289603 (2007j:53070)

[2] M. T. Anderson, Remarks on Perelman's papers, preprint, available at http://www.math. sunysb.edu/ anderson/perelman.pdf.

[3] A. L. Besse, Einstein manifolds, Ergebnisse der Math. Springer-Verlag, Berlin-New York, 1987. MR867684 (88f:53087) 
[4] H. D. Cao, Deformation of Kähler metrics on compact Kähler manifolds, Invent. Math. 81 (1985), 359-372. MR799272 (87d:58051)

[5] F. Fang, Y. Zhang, Perelman's $\lambda$-functional and the Seiberg-Witten equations, Frontiers of Mathematics in China, 2, no. 2 (2007), 191-210. MR2299748 (2008e:53051)

[6] F. Fang, Y. Zhang and Z. L. Zhang, Non-singular solutions to the normalized Ricci flow equation, Math. Ann. 340 (2008), 647-674. MR2357999 (2009a:53112)

[7] F. Fang, Y. Zhang and Z. L. Zhang, Non-singular solutions of normalized Ricci flow on noncompact manifolds of finite volume, Journal of Geometric Analysis, 20, no. 3 (2010), 592-608. MR2610891

[8] R. Hamilton, Three-manifolds with positive Ricci curvature, J. Diff. Geom. 17 (1982), 255306. MR664497 (84a:53050)

[9] R. Hamilton, Non-singular solutions of the Ricci flow on three-manifolds, Commun. Anal. Geom. 7 (1999), 695-729. MR.1714939 (2000g:53034)

[10] N. J. Hitchin, On compact four-dimensional Einstein manifolds, J. Differential Geom. 9 (1974), 435-442. MR0350657 (50:3149)

[11] M. Ishida, The normalized Ricci flow on four-manifolds and exotic smooth structure, arXiv:math.0807.2169.

[12] B. Kleiner and J. Lott, Notes on Perelman's papers, Geom. Topol. 12 (2008), 2587-2855. MR2460872 (2010h:53098)

[13] D. Kotschick, Monopole classes and Perelman's invariant of four-manifolds, arXiv:math. DG/0608504.

[14] C. LeBrun, Kodaira dimension and the Yamabe problem, Comm. Anal. Geom. 7 (1999), 133-156. MR1674105 (99m:58056)

[15] G. Perelman, The entropy formula for the Ricci flow and its geometric applications, arXiv:math.DG/0211159.

[16] G. Perelman, Ricci flow with surgery on three-manifolds, arXiv:math.DG/0303109.

[17] O. S. Rothaus, Logarithmic Sobolev inequalities and the spectrum of Schrödinger operators, J. Funct. Anal. 42 (1981), 110-120. MR620582 (83f:58080b)

[18] J. Song, G. Tian, The Kähler-Ricci flow on surfaces of positive Kodaira dimension, Inventiones Mathematicae 170, no. 3 (2007), 609-653. MR2357504 (2008m:32044)

[19] H. Tsuji, Existence and degeneration of Kähler-Einstein metrics on minimal algebraic varieties of general type, Math. Ann. 281 (1988), 123-133. MR944606 (89e:53075)

[20] G. Tian, Z. Zhang, On the Kähler-Ricci flow on projective manifolds of general type, Chinese Annals of Mathematics B 27 (2006), 179-192. MR2243679 (2007c:32029)

[21] Y. Zhang, Miyaoka-Yau inequality for minimal projective manifolds of general type, Proc. Amer. Math. Soc. 137 (2009), 2749-2754. MR2497488 (2010b:32036)

[22] Z. L. Zhang, Compact blow-up limits of finite time singularities of Ricci flow are shrinking Ricci solitons, C. R. Acad. Sci. Paris, Ser. I 345 (2007), 503-506. MR2375111(2009a:53117)

[23] Z. Zhang, Scalar curvature bound for Kähler-Ricci flows over minimal manifolds of general type, International Mathematics Research Notices (2009), 3901-3912. MR2544732 (2010j:32038)

Department of Mathematics, Capital Normal University, Beijing, People's Republic OF CHINA

E-mail address: yuguangzhang76@yahoo.com

Department of Mathematics, Capital Normal University, Beijing, People's Republic OF CHINA

E-mail address: zhleigo@yahoo.com.cn 